\title{
Thomas-Fermi screening of a moving surface charge
}

I. O. Kulik

Citation: Low Temperature Physics 23, 650 (1997);

View online: https://doi.org/10.1063/1.593473

View Table of Contents: http://aip.scitation.org/toc/ltp/23/8

Published by the American Institute of Physics

\section{Articles you may be interested in}

Generalized charge-screening in relativistic Thomas-Fermi model

Physics of Plasmas 21, 102702 (2014); 10.1063/1.4897320

La-doped $\mathrm{SrTiO}_{3}$ films with large cryogenic thermoelectric power factors

Applied Physics Letters 102, 182101 (2013); 10.1063/1.4804182

In-situ probing of coupled atomic restructuring and metallicity of oxide heterointerfaces induced by polar adsorbates

Applied Physics Letters 111, 141604 (2017); 10.1063/1.4991956

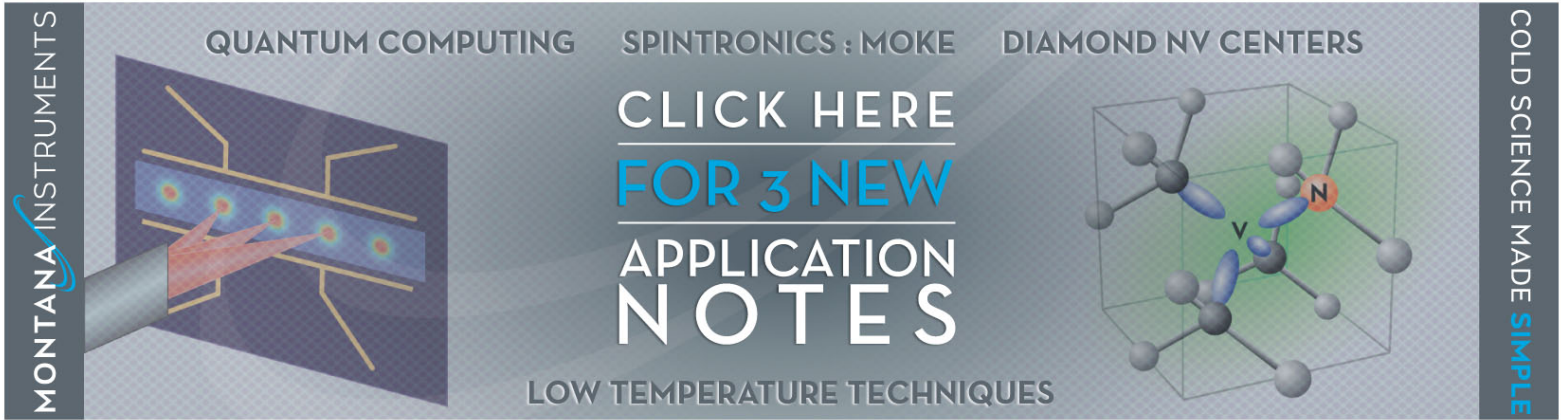




\title{
Thomas-Fermi screening of a moving surface charge
}

\author{
I. O. Kulik
}

Department of Physics, Bilkent University, Bilkent 06533, Ankara, Turkey and B. Verkin Institute for Low Temperature Physics and Engineering, National Academy of Sciences of Ukraine, 47 Lenin ave., Kharkov 310164, Ukraine

(Submitted February 18, 1997)

Fiz. Nizk. Temp. 23, 864-879 (August 1997)

The dynamical screening effects in the skin layer of a metal are investigated. The electric charge density near the metal surface induced by a moving charged body outside the metal is screened at the Thomas-Fermi length if the velocity parallel to the surface is smaller than the Fermi velocity. Crisis of screening is found at the velocity approaching the Fermi velocity, which results in the electric field penetration inside the metal at large distances, and in the distortion of the electric field distribution outside the metal. The energy dissipation from a moving charged body as a function of the velocity has a pronounced singularity near the Fermi velocity. (C) 1997 American Institute of Physics. [S1063-777X(97)01208-5]

\section{INTRODUCTION}

Macroscopic charge cannot exist inside a metal. Upon introduction into a metallic sample, any external charge concentrates near its surface in a thin layer, whose characteristic thickness is ${ }^{1,2}$

$$
\lambda_{T F}=\left[4 \pi e^{2} N\left(\varepsilon_{F}\right)\right]^{-1 / 2},
$$

the so-called Thomas-Fermi screening length, which is typically of the order of a few angstroms. $\left[N\left(\varepsilon_{F}\right)\right.$ is the density of electronic states at the Fermi energy, $\varepsilon_{F}$.]

If the external charge is fixed in space, the emerging Coulomb potential will be screened inside the metal at the same distance. Along the surface, charge density can be localized within some area, and can be translated parallel to the surface without changing its shape. It is tempting to consider the surface charge, which is generated due to the motion of a charged body in vacuum near the metal surface, as a separate entity, and to investigate the effects related to its dynamical behavior. At the velocity smaller than the Fermi velocity, the nonlinearity in the response to an external perturbation may occur if the former approaches the phonon propagation velocity, which results in phonon emission followed by extra energy release from the surface sheet. In the case of fast motion with a velocity greater than the Fermi velocity, the oscillatory potential emerges in the wake behind the charged body (e.g., an ion moving in a metal), which can trap conduction electrons in the wake-bound state. ${ }^{3,4}$ At a velocity approaching the Fermi velocity, the charged body wake is at "resonance", with the conduction electrons, which accounts for the singularity of the dissipation in the surface sheet and for the stopping power of body motion. In the case of motion of a charged body outside the metal, this results in the nonlinear interaction between the external moving charge and the induced charge near the surface. The dependence of drag force and power dissipation on the velocity is nonlinear and possibly nonmonotonic.

The information concerning the electron states in metal, which can be obtained in the corresponding experiments, is similar to that found from the conventional conductivity measurements except that (1) it is directly related to the re- laxation processes and mechanisms very near the metal surface; (2) the nonlinear output is expected in the linear amplitude regime (small charges and fields) since the nonlinearity may be concerned with the large velocity of collective motion rather than with the drift velocity of electrons.

In the present paper we investigate the dependence of the charge distribution inside the metal and the electrostatic potential outside the metal, on the velocity of the surface sheet motion produced by a charged body (known as the "tip") outside the metal moving parallel to the metal surface. It is shown that the surface charge follows the tip motion adiabatically only if the velocity of motion is much smaller than the Fermi velocity $V_{F}$. A velocity greater than $V_{F}$ causes a crisis of the Thomas-Fermi screening, which results in the nonlinear charge penetration deep into the metal and in the distortion of the screening electric field inside and outside the metal.

The questions considered can have relevance to scanning tunneling microscopy, ${ }^{5}$ to the effects of charge quantization in small metallic electrodes, ${ }^{6}$ to ballistic electron transport in narrow metallic constrictions and point contacts, ${ }^{7,8}$ and to general aspects of "fermiology," i.e., Fermi surface reconstruction in metals, since the dynamical screening effects in the surface sheet depend essentially on the topology and shape of the Fermi surface. The interaction of a moving surface charge with phonons can be viewed as a kind of "surface spectroscopy", of conduction electrons in metals. ${ }^{9}$

Another type of experiment involves charged ion motion inside a metal ${ }^{3}$ or a traversal of the interface between metal and vacuum. ${ }^{4}$ If the velocity of ion motion approaches $V_{F}$ from above, the wake-bound state of an electron and stopping power for ion motion reveal a singularity in the limit $V \rightarrow V_{F}$. In the case of small velocity, the surface charge follows the external perturbation adiabatically, allowing for a semiclassical description of the interaction of external electric field and the induced charge. Important difference between the case $V \gg V_{F}$ and $V \leqslant V_{F}$ is that semiclassical approximation may present a reasonable approximation of the problem.

After the discussion of the validity of different approxi- 
mations (semiclassical or random-phase), which are applicable to the problem of dynamical screening in Sec. 2, we investigate in Sec. 3 the dynamical screening in a twodimensional metal with a cylindrical Fermi surface since it most clearly illustrates the theoretical method adopted by us and the origin of the velocity-dependent anomaly predicted. In Sec. 4, similar effects are considered for a threedimensional metal with a spherical Fermi surface. Energy dissipation and drag force induced in a moving body are calculated in Sec. 5, followed in Sec. 6 by the discussion of the physical aspects of the surface charge dynamics and possible realization of its fast motion in metals.

\section{SEMICLASSICAL APPROXIMATION FOR A DYNAMICAL SCREENING}

Linear response of a degenerate electron gas to a timeand space-dependent electric potential

$$
\phi(\mathbf{r}, t)=\int \frac{d \mathbf{k}}{(2 \pi)^{3}} \int_{-\infty}^{\infty} \frac{d \omega}{2 \pi} \phi_{\mathbf{k} \omega} \exp (i \mathbf{k r}-i \omega t)
$$

is described by the quantum kinetic equation ${ }^{10}$ (assuming $\hbar$ $=1)$

$$
\left(\omega-\varepsilon_{\mathbf{p}+\mathbf{k} / 2}+\varepsilon_{\mathbf{p}-\mathbf{k} / 2}\right) f_{\mathbf{k} \omega}^{1}+e \phi_{\mathbf{k} \omega}\left(f_{\mathbf{p}-\mathbf{k} / 2}^{0}-f_{\mathbf{p}+\mathbf{k} / 2}^{0}\right)=0,
$$

where $f_{\mathbf{p}}^{0}$ is the unperturbed electron distribution function $\left(\exp \left[\left(\varepsilon_{\mathbf{p}}-\mu\right) / T\right]+1\right)^{-1}$, and $f_{\mathbf{k} \omega}^{1}$ is the first order correction to $f_{\mathbf{k} \omega}(\mathbf{p})$. (Assuming that the velocity of motion is much less than the light velocity $c$, we can ignore the magnetic field effects and eliminate the vector potential A, leaving only a scalar potential $\phi$.)

Equation (2) results in the Lindhard formula (e.g., see Ref. 1) for the relation between the electric displacement and the electric field

$$
\mathbf{D}_{\mathbf{k} \omega}=\mathbf{E}_{\mathbf{k} \omega}+4 \pi \mathbf{P}_{\mathbf{k} \omega}=\epsilon(\mathbf{k}, \omega) \mathbf{E}_{\mathbf{k} \omega},
$$

where $\rho_{\mathbf{k} \omega}=-(4 \pi)^{-1} i \mathbf{k} \mathbf{P}_{\mathbf{k} \omega}$ is the external charge density, and

$$
\varepsilon(\mathbf{k}, \omega)=1+\frac{4 \pi e^{2}}{\mathbf{k}^{2}} \int \frac{2 d \mathbf{p}}{(2 \pi)^{3}} \frac{f_{\mathbf{p}+\mathbf{k} / 2}^{0}-f_{\mathbf{p}-\mathbf{k} / 2}^{0}}{\omega-\varepsilon_{\mathbf{p}+\mathbf{k} / 2}+\varepsilon_{\mathbf{p}-\mathbf{k} / 2}-i \delta} .
$$

At $\omega=0$, the dielectric function within the random-phase approximation (RPA) [Eq. (2)] is

$$
\epsilon(\mathbf{k})=1+\frac{\kappa_{T F}^{2}}{k^{2}} L(x), \quad L(x)=\frac{1}{2}+\frac{1-x^{2}}{4 x} \ln \left|\frac{1+x}{1-x}\right|,
$$

where $x=2 k / k_{F}$. At small $\mathbf{k}$, the kinetic equation (2) reduces to a semiclassical (SA) Boltzmann kinetic equation for the distribution function $f(\mathbf{p}, \mathbf{r}, t)$, and Eq. (3) reduces to an expression for the dielectric function

$$
\epsilon(\mathbf{k})=1+\kappa_{T F}^{2} / k^{2},
$$

which is equivalent to (1) with $\kappa_{T F}=1 / \lambda_{T F}$.

To clarify the difference between various approximations, let us consider the screening of the electrostatic potential produced by a charged plane immersed inside the metal.
The scalar potential in a metal emerging from an external electric charge uniformly distributed with the density $\sigma$ in a plane $z=0$ is

$$
\phi(z)=2 \sigma \int_{-\infty}^{\infty} \frac{\exp (i k z)}{k^{2} \epsilon(\mathbf{k})} d k .
$$

It reduces to an exponential dependence $\phi(z)$ $=\phi(0) \exp \left(-\kappa_{T F}|z|\right)$ within the SA. Within the RPA, by introducing a parameter

$$
\alpha=\left(\frac{\kappa_{T F}}{2 k_{F}}\right)^{2}
$$

we obtain

$$
\phi(z)=\int_{0}^{\infty} \frac{\cos \left(2 k_{F} z x\right)}{x^{2}+\alpha f(x)} d x .
$$

For typical metals, $\alpha$ falls within the interval

$$
0.3<\alpha<1
$$

[ $\alpha$ is related to the most commonly used quantity ${ }^{2} r_{s}$ $=r_{0} / a_{0}$, where $a_{0}$ is the Bohr radius, and $r_{0}$ is the average distance between electrons, since $\alpha=\pi^{-1}(4 / 9 \pi)^{1 / 3} r_{s}$ $\left.=0.1659 r_{s}.\right]$

The normalized potential distribution $\phi(z) / \phi(0)$ as a function of $2 k_{F} z$ is shown in Fig. 1a for various $\alpha$. However, since it is nonexponential (power-like and oscillating with a period $\pi / k_{F}{ }^{1}$ at large $\left.z\right), \phi(z)$ is very small in the region in which, within the SA, it decays exponentially. If replotted as a function of $\kappa_{T F} z=z / \lambda_{T F}$, all the dependences $\phi(z) / \phi(0)$ at different $\alpha$ fall nearly into a single line (Fig. 1b). The screening radius,

$$
\bar{r}=\int_{0}^{\infty} \phi(z) d z / \phi(0),
$$

within $10 \%$ accuracy equals the Thomas-Fermi screening length in the interval of $\alpha$ from 0 to 1 . This has an implication that the semiclassical approximation, which is not exact, nevertheless gives a reasonable estimate of screening. We will use the approximation which can be used to trace the dynamical screening effects in metals. The solution proves to be quite complex even within the SA, and it would become intractable in the RPA scheme ${ }^{11}$ since $k$ in Eq. (2) must be considered as an operator $i d / d z$. In any case, the validity of $\mathrm{SA}$ is indeed guaranteed as long as $\alpha$ is small (9).

\section{THOMAS-FERMI SCREENING IN A TWO-DIMENSIONAL METAL}

Consider the metallic semispace in the vicinity of a charged tip $T$ moving parallel to the metal surface with a velocity $V$ (Fig. 2). We shall investigate the steady-state distribution of electrons in a momentum space $f(\mathbf{r}, \mathbf{p}, t)$ and the electrostatic potential distribution $\phi(\mathbf{r}, t)$ inside and outside the metal with the assumption that they make a self-similar configuration which depends on the relative coordinate $x$ $-V t$.

In a semiclassical approximation, charge density $\rho$ is expressed in terms of $f$ as 

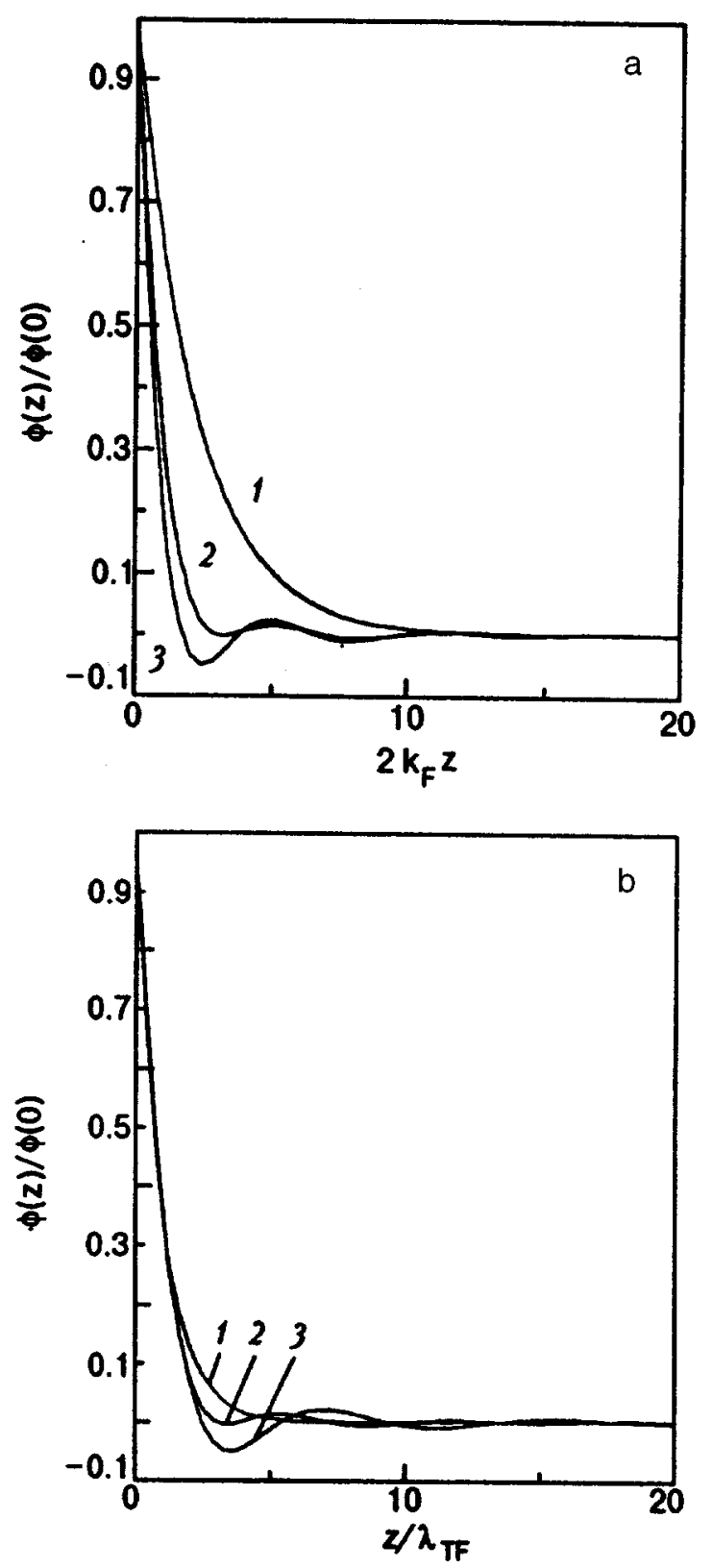

FIG. 1. Normalized potential distribution inside a metal at various values of $\alpha$ as a function of $2 k_{F} z$ (a) and $\kappa_{T F} z$ (b). $1-\alpha=0.2 ; 2-\alpha=1.1 ; 3-\alpha$ $=2.0$.

$$
\rho=2 e \int \frac{d \mathbf{p}}{(2 \pi \hbar)^{3}}\left(f-f_{0}\right),
$$

where $f_{0}$ is the equilibrium Fermi distribution. The scalar potential can be found from the Poisson equation

$$
\nabla^{2} \phi+4 \pi \rho=0,
$$

and $f$ satisfies the Boltzmann equation

$$
\frac{\partial f}{\partial t}+\mathbf{v} \frac{\partial f}{\partial \mathbf{r}}-e \nabla \phi \frac{\partial f}{\partial \mathbf{p}}=-\hat{\mathbf{v}}\left(f-f_{0}\right),
$$

in which $\hat{\mathrm{v}}$ is an electron collision operator. The self-similar distributions of $f$ and $\phi$ are

$$
f=f_{0}+\chi_{\varphi}(x-V t, y, \mathbf{p}) \partial f_{0} / \partial \varepsilon_{\mathbf{p}}, \quad \phi=\phi(x-V t) .
$$

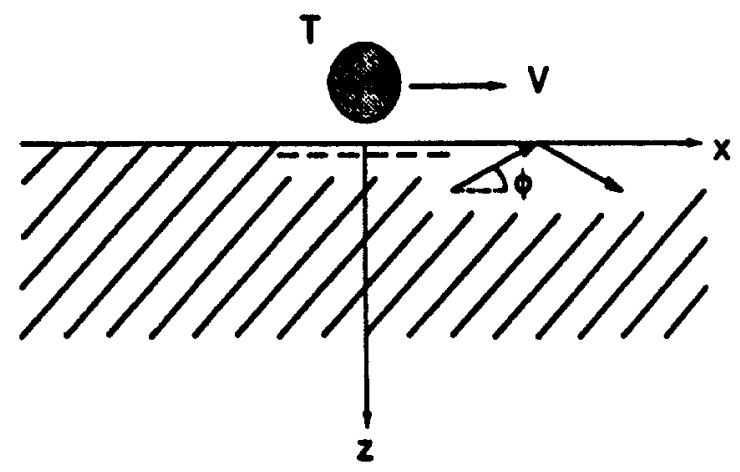

FIG. 2. Schematic diagram of a charged tip ( $T$ ) moving parallel to the metal surface with a velocity $V$. Surface charge (a dashed line) accumulates near the metal surface and moves with the same velocity. $\varphi$ is the angle of incidence of the electron.

The charge density in a metal at $T=0$ is

$$
\rho=-e N\left(\varepsilon_{F}\right)\left\langle\chi_{\varphi}\right\rangle,
$$

where $\langle\ldots .$.$\rangle denotes averaging over the Fermi surface.$

We ignore scattering of electrons inside a metal, which is expected to be a good approximation if the electron mean free path is much larger than the Thomas-Fermi screening length, but include the scattering of electrons at the surface with the help of the diffuse boundary condition that introduces a diffusivity coefficient $q(0<q<1)$. Requiring that the electron current be zero at the metal surface, ${ }^{12}$ we can write the boundary condition in case of a cylindrical Fermi surface directed along the $y$ axis in the form

$$
\chi_{-\varphi}=(1-q) \chi_{\varphi}+\frac{q}{2} \int_{0}^{\pi} \chi_{\varphi} \sin \varphi d \varphi,
$$

where $q$ is the diffusivity coefficient of the metal surface, and $\varphi$ is the angle between the direction of electron momentum and surface.

In the Fourier representation with respect to the surface coordinates $x, y$, the equations for $\phi_{k}$ and $\chi_{\varphi k}$ are (below we drop for clarity the index $\mathbf{k}$ )

$$
k^{2} \phi-d^{2} \phi / d z^{2}=-4 \pi e N\left(\varepsilon_{F}\right)\left\langle\chi_{\varphi}\right\rangle
$$

and

$$
\begin{gathered}
{\left[\nu+i k_{x}\left(V_{F} \cos \varphi-V\right)\right] \chi_{\varphi}+V_{F} \sin \varphi \frac{d \chi_{\varphi}}{d z}} \\
=e V_{F}\left(i k_{x} \cos \varphi+\sin \varphi \frac{d}{d z}\right) \phi .
\end{gathered}
$$

Although we are considering a clean metal (collision frequency $\mathrm{v} \rightarrow 0)$, a "trace" of the electron scattering $(v=+0)$ should remain in order to ensure a proper analytical behavior of the electron distribution inside a metal as $z \rightarrow \infty$.

In the case of zero velocity, $V=0$, Eq. (18) gives $\chi_{\varphi}$ $=e \phi$, thus resulting in an exponential distribution of $\phi$ inside the metal

$$
\phi=\phi(0) \exp \left(-\kappa_{T F} z\right) \text { with } \kappa_{T F}=\sqrt{\lambda_{T F}^{-2}+k_{x}^{2}} .
$$


We shall use below the dimensionless units such that $\hbar$ $=1, e=1$ and $N\left(\varepsilon_{F}\right)=1, V_{F}=1$, where $V_{F}$ is the Fermi velocity. Thus, representing $\chi_{\varphi}$ in the form $\chi_{\varphi}=\phi$ $+u_{\varphi}$, we obtain

$$
\left(-k_{x}^{2}+\frac{d^{2}}{d z^{2}}\right) \phi=\phi+\left\langle u_{\varphi}\right\rangle
$$

and

$$
\left(i \gamma_{\varphi}+\frac{d}{d z}\right) u_{\varphi}=\frac{i k_{x} V}{\sin \varphi} \phi
$$

where

$$
\gamma_{\varphi}=\frac{k(\cos \varphi-V)-i \mathrm{v}}{\sin \varphi}, \quad \mathrm{v}=+0 .
$$

The solution of Eq. (21) is

$$
\begin{aligned}
u_{\varphi}= & A_{\varphi} \exp \left(-i \gamma_{\varphi}\right)+\frac{i k_{x} V}{\sin \varphi} \int_{0}^{z} \phi\left(z^{\prime}\right) \exp \left[-i \gamma_{\varphi}(z\right. \\
& \left.\left.-z^{\prime}\right)\right] d z^{\prime},
\end{aligned}
$$

from which it follows that $\phi(z)$ can be obtained with the Laplace transform

$$
\phi_{p}=p \int_{0}^{\infty} \phi(z) \exp (-p z) d z
$$

giving for the space dependence of $\phi$ at $z>0$

$$
\begin{aligned}
\phi(z)= & \frac{1}{2 \pi i} \int_{a-i \infty}^{a+i \infty} d p \mathrm{e}^{p z} \\
& \times \frac{p \phi(0)+\phi^{\prime}(0)+\int_{-\pi}^{\pi}(d \varphi / 2 \pi) A_{\varphi} /\left(p+i \gamma_{\varphi}\right)}{p^{2}-k_{x}^{2}-S(p)}
\end{aligned}
$$

where $S(p)$ is a function

$$
S(p)=1+k_{x} V \int_{-\pi}^{\pi} \frac{d \varphi}{2 \pi} \frac{1}{k_{x}(\cos \varphi-V-i 0)-i p \sin \varphi} .
$$

Integral (24) is taken in a complex plane $p$ along a vertical line which is situated to the right of all singularities (poles and branching lines) of the integrand (Fig. 3). The solution depends upon the analytical properties of $S(p)$ which will be discussed below, and is different at $V<1$ (velocity smaller than the Fermi velocity) and at $V>1$.

The requirement that $\phi(z)$ derived from (24) behaves regularly at $z \rightarrow \infty$ establishes the relation between $\phi(0)$ and $\phi^{\prime}(0)$ (prime denotes derivative with respect to $z$ ) and thus allows the solution of the Poisson equation outside the metal, which for clarity we also represent in the form of a Poisson integral:

$$
\begin{aligned}
& \phi=\frac{1}{2 \pi i} \int_{a-i \infty}^{a+i \infty} d p e^{-p z} \frac{p \phi(0)-\phi^{\prime}(0)-4 \pi Q e^{-p h}}{p^{2}-k_{x}^{2}}, \\
& z<0,
\end{aligned}
$$

where for simplicity it is assumed that the tip is a point charge $Q$ located at a height $h$ above the metal surface. Re-

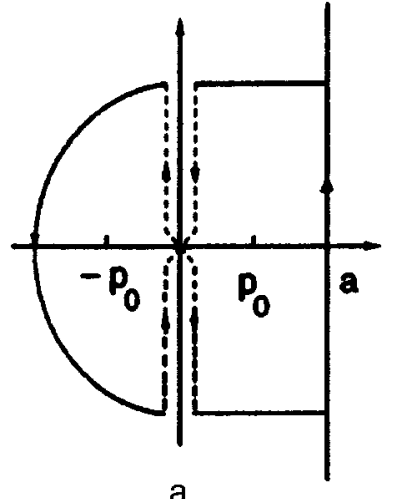

a

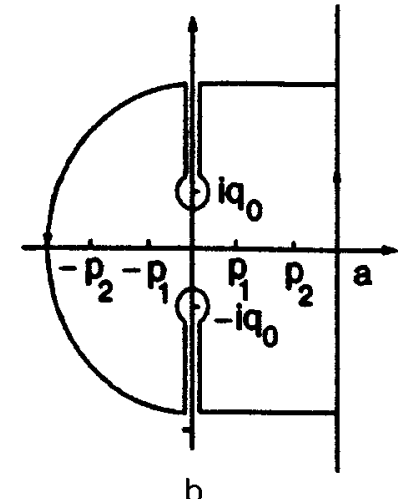

b
FIG. 3. Path of integration in Eq. (24) for $V<1$ (a) and $V>1$ (b). Integrals along broken lines cancel each other because $S(p)$ at $V<1$ has the same value on both sides to the left and to the right of the imaginary axis.

quirement that $\phi(z)$ in (24) should properly behave at $z \rightarrow$ $-\infty$ allows us to find the potential provided that the value of the ratio $\phi^{\prime}(0) / \phi(0)$ is specified by the solution of the Poisson equation inside the metal. ${ }^{13}$

Evaluation of the integral (24) at $V<1$ gives

$$
\phi(z)=\phi(0) \mathrm{e}^{-p_{0} z}+\int_{-\pi}^{\pi} \frac{\mathrm{e}^{-p_{0} z}-\mathrm{e}^{-i \gamma_{\phi} z}}{p_{0}^{2}+\gamma_{\varphi}^{2}} A_{\varphi},
$$

where $\phi^{\prime}(0)$ is related to $\phi(0)$ according to

$$
\phi^{\prime}=-p_{0} \phi(0)-\int_{-\pi}^{\pi} \frac{d \varphi}{2 \pi} \frac{A_{\varphi}}{p_{0}+i \gamma_{\varphi}} .
$$

This is a consequence of the vanishing $\exp \left(p_{0} z\right)$ terms in $\phi(z)$, where $p_{0}$ is the pole of the denominator of the integrand of Eq. (24).

Substitution of Eq. (27) into (23) gives

$$
\begin{aligned}
u_{\varphi}= & A_{\varphi} \exp \left(-i \gamma_{\varphi}\right)+\frac{k_{x} V}{\sin \varphi}\left[\frac { \phi _ { 0 } } { \gamma _ { \varphi } + i p _ { 0 } } \left[\exp \left(-p_{0} z\right)\right.\right. \\
& \left.-\exp \left(-i \gamma_{\varphi} z\right)\right] \\
& \left.+\int_{-\pi}^{\pi} \frac{d \varphi}{2 \pi} \frac{A_{\varphi^{\prime}}}{p_{0}^{2}+\gamma_{\varphi^{\prime}}^{2}} \frac{\exp \left(-i \gamma_{\varphi^{\prime}} z\right)-\exp \left(-i \gamma_{\varphi^{\prime}} z\right)}{\gamma_{\varphi}-\gamma_{\varphi^{\prime}}}\right],
\end{aligned}
$$

where

$$
\phi_{0}=\phi(0)+\int_{-\pi}^{\pi} \frac{d \varphi}{2 \pi} \frac{A_{\varphi}}{p_{0}^{2}+\gamma_{\varphi}^{2}} .
$$

The positive values of $\varphi(0<\varphi<\pi)$ correspond to electrons reflected from the surface and the negative values of $\varphi(-\pi<\varphi<0)$ correspond to electrons arriving from the bulk of the metal. The quantity $A_{\varphi}$ in Eq. (29) satisfies at $\varphi<0$ the same relation (16) as $\chi_{\varphi}$ does. For positive $\varphi$, the exponents $\exp \left(-i \gamma_{\varphi}\right)$ taken with the finite value of $\mathrm{v}$ increase exponentially inside the metal and therefore should cancel themselves out. This condition gives the relation, which is valid at $-\pi<\varphi<0$ : 


$$
\begin{aligned}
A_{\varphi}= & \frac{k_{x} V}{\sin \varphi}\left[\frac{\phi_{0}}{\gamma_{\varphi}+i p_{0}}\right. \\
& \left.-\int_{-\pi}^{\pi} \frac{d \varphi^{\prime}}{2 \pi} \frac{A_{\varphi^{\prime}}}{\left(p_{0}^{2}+\gamma_{\varphi^{\prime}}^{2}\right)\left(\gamma_{\varphi}-\gamma_{\varphi^{\prime}}\right)}\right] .
\end{aligned}
$$

This relation closes the set of equations necessary for the determination of the field distribution inside the metal. Combination of Eq. (24) with the boundary condition for $A_{\varphi}$ results in an integral equation for $A_{\varphi}$ in the domain $0<\varphi$ $<\pi$

$$
\begin{aligned}
& \hat{L} A_{\varphi}+\frac{k_{x} V}{\sin \varphi} \int_{0}^{\pi} \frac{d \varphi^{\prime}}{2 \pi} \frac{1}{p_{0}^{2}+\gamma_{\varphi^{\prime}}^{2}}\left(\frac{A_{\varphi^{\prime}}}{\gamma_{\varphi}+\gamma_{\varphi^{\prime}}}+\frac{\hat{L} A_{\varphi^{\prime}}}{\gamma_{\varphi}-\gamma_{\varphi^{\prime}}}\right) \\
& =\frac{k_{x} V}{\sin \varphi} \frac{\phi_{0}}{\gamma_{\varphi}-i p_{0}},
\end{aligned}
$$

where $\phi_{0}$ is taken from Eq. (30), and $\hat{L}$ is the operator of diffusive reflection

$$
\hat{L} A_{\varphi}=(1-q) A_{\varphi}+\frac{q}{2} \int_{0}^{\pi} A_{\varphi} \sin \varphi d \varphi .
$$

Once solved, Eq. (32) can be used to find the ratio $\phi^{\prime} / \phi$ at the metal surface, which is our goal in solving selfconsistently for the field distribution inside and outside the metal.

Let us evaluate $p_{0}$ and $S(p)$. Consider separately the cases $V<1$ and $V>1$.

Expression (25) can be reduced to an integral along the unit circle $z=\exp (i \varphi)$ in the complex plane $z$,

$$
S(p)=1+\oint \frac{d z}{2 \pi i} \frac{2 k_{x} V}{\left(k_{x}-p\right) z^{2}-2\left(k_{x} V+i 0\right) z+k_{x}+p} .
$$

At $V<1$, the poles of the denominator in the integrand,

$$
z_{1,2}=\left(k_{x} V+i \mathrm{v} \pm \sqrt{\left(k_{x} V+i \mathrm{v}\right)^{2}+p^{2}-k_{x}^{2}}\right) /\left(k_{x}-p\right),
$$

lie either inside or outside the unit circle and therefore the integral is equal to zero (except for $\operatorname{Re} p=0$ ). We therefore have

$$
S(p)=1+\frac{i}{\left(1-V^{2}\right)^{1 / 2}} \frac{\delta(p)}{\delta(0)}, \quad V<1 .
$$

The poles of the denominator of the integrand in Eq. (24) are $\pm p_{0}$, where

$$
p_{0}=\sqrt{1+\mathbf{k}^{2}} \text {. }
$$

Typical values of $|\mathbf{k}|$ are of the order of the inverse distance from the tip to the metal surface, which is assumed to be much larger than the Thomas-Fermi screening length $\lambda_{T F}$, and therefore $|\mathbf{k}|$ is much smaller than the characteristic momentum $\kappa_{T F}\left[\kappa_{T F}=(4 \pi)^{1 / 2}\right.$ in dimensionless units].

In the case $V>1$, the behavior of $S(p)$ is quite different. At the real axis $S(p)$ is

$$
S(p)=1-\frac{\left|k_{x}\right| V}{\left[p^{2}+k_{x}^{2}\left(V^{2}-1\right)\right]^{1 / 2}}, \quad V>1 .
$$

This function has branching points at the imaginary axis $p$ $= \pm i q_{0}$, where $q_{0}=k_{x}\left(V^{2} s-1\right)^{1 / 2}$. At the real axis, the denominator of the integrand of Eq. (24) has two pairs of poles $\pm p_{1}$ and $\pm p_{2}$. For example, in the case $k_{y}=0$ the equation for the poles

$$
p^{2}=k_{x}^{2}+1-\frac{\left|k_{x}\right| V}{\left[p^{2}+k_{x}^{2}\left(V^{2}-1\right)\right]^{1 / 2}}
$$

gives two values for $p>0$ :

$$
p=p_{1}=k_{x}, \quad \text { and } p=p_{2}=1-\frac{1}{2} k_{x} V,\left|k_{x}\right| \ll 1 \text {. }
$$

The first pole signals that the electric field distribution breaks the Thomas-Fermi barrier and penetrates into a metal to distances $\left|k_{x}\right|^{-1}$ of the order of the tip-to-surface distance, which is much larger than $\lambda_{T F}$. This, however, is not an equilibrium charge distribution.

With the two poles $p_{1,2}$, the potential $\phi(z)$, which is derived from Eq. (24) by integration along the contour shown in Fig. $3 b$ has two exponentially increasing terms $\exp \left(p_{1} z\right)$ and $\exp \left(p_{2} z\right)$, and also the nonsingular terms exp $\left(-p_{1} z\right), \exp \left(-p_{2} z\right), \exp \left( \pm i q_{0} z\right)$, and $\exp \left(-i \gamma_{\varphi} z\right)$, where $q_{0}=k_{x}\left(V^{2}-1\right)^{1 / 2}$. Elimination of singular contributions results in the number of equations which is larger than the number of variables. This means that the only admissible solution in this case is a trivial one, $A_{\varphi}=0, u_{\varphi}=0, \phi_{0}$ $=0$. We thus find that $\phi(0)=\phi^{\prime}(0)=0$, which is inconsistent with the equation for the potential value outside the metal [Eq. (26)]. In fact, if $\phi(0)=\phi^{\prime}(0)=0$ (note that these quantities are functions of $\mathbf{k}$ ) in some domain of $\mathbf{k}$, then in this same domain the potential will become infinite at large $z$. We conclude, therefore, that there is no regular solution for $\phi(z)$ if the velocity of the tip $V$ is greater than the Fermi velocity.

This means that the solution $\phi(z)$ does not exist in the linear approximation in $\chi_{\varphi}$, and higher-order terms in the electron distribution should be taken into account on the right side of the Poisson equation (17).

\section{DYNAMICAL SCREENING IN A THREE-DIMENSIONAL METAL}

It can be assumed that the instability of the steady-state motion of a surface sheet at high velocity found in the previous section is specific to the two-dimensional Fermi surface. We shall see, however, that similar property is also seen in a three-dimensional metal.

In a metal with a spherical Fermi surface, an equation for the angular-dependent part of the electron distribution analogous to (21) is

$$
\left(i \gamma_{\varphi}+\frac{d}{d z}\right) u_{\varphi}=\frac{i k_{x} V}{\sin \theta \sin \varphi} \phi
$$

where $\theta$ is a polar angle of the electron momentum at the Fermi surface, and $\gamma_{\varphi}$ is a quantity

$$
\gamma_{\varphi}=\frac{k_{x}(\sin \theta \cos \varphi-V)+k_{y} \cos \theta-i \mathrm{~V}}{\sin \theta \sin \varphi} .
$$

The boundary condition of diffuse scattering at $z=0$ and $0<\varphi<\pi$ is 

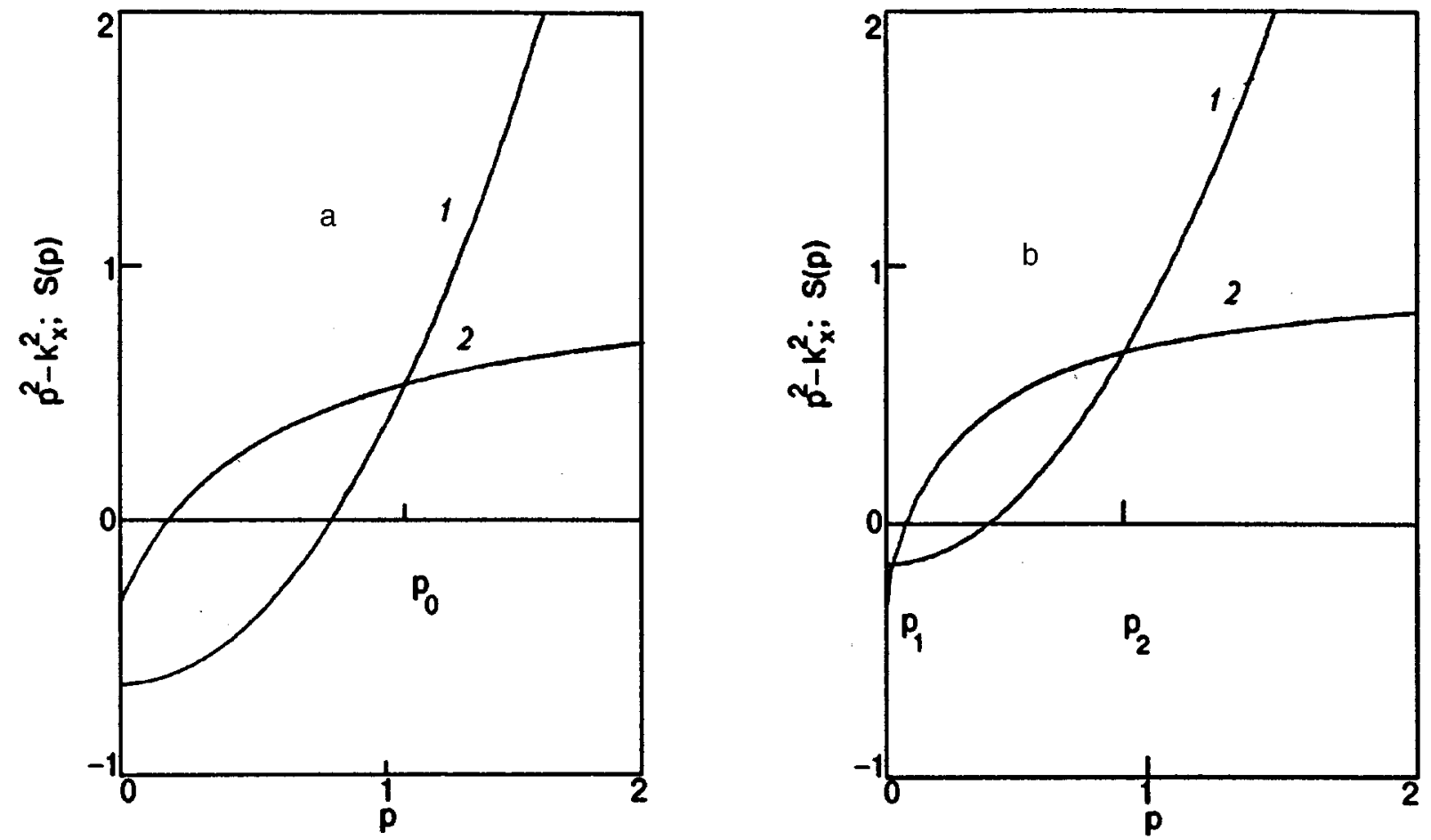

FIG. 4. Poles of the denominator in Eq. (44) at $k_{y}=0$ and $V=0.9$. (a) large value of $k_{x}\left(k_{x}=0.8\right)$ corresponding to one pole $p_{0}$; (b) small $k_{x}\left(k_{x}\right.$ $=0.4$, two poles $\left.-p_{1}, p_{2}\right)$. Curve 1 - the dependence $p^{2}-k_{x}^{2}$, curve 2 - the dependence $S(p)$.

$$
u_{-\varphi}=(1-q) u_{\varphi}+\frac{q}{\pi} \int_{0}^{\pi} \sin \theta d \theta \int_{0}^{\pi} d \varphi u_{\varphi} \sin \theta \sin \varphi
$$

The dynamical screening is represented by a threedimensional $S$-function analogous to $(25)$

$$
\begin{aligned}
S= & 1+\int \frac{d \Omega}{4 \pi} \\
& \times \frac{k_{x} V}{k_{x}(\sin \theta \cos \varphi-V)+k_{y} \cos \theta-i p \sin \theta \sin \varphi-i \mathrm{~V}},
\end{aligned}
$$

where $d \Omega=\sin \theta d \theta d \varphi$, which gives the potential distribution

$$
\begin{aligned}
\phi(z)= & \frac{1}{2 \pi i} \int_{a-i \infty}^{a+i \infty} d p \mathrm{e}^{p z} \\
& \times \frac{\phi(0)+p \phi^{\prime}(0)+\int(d \Omega / 4 \pi)\left[A_{\varphi} /\left(p+i \gamma_{\varphi}\right)\right]}{p^{2}-\mathbf{k}^{2}-S(p)} .
\end{aligned}
$$

Evaluation of an integral (43) at $k_{y}=0$ and $V<1$ gives
$S=$

$$
\left\{\begin{array}{l}
1-\frac{V}{\left(1-p^{2} / k_{x}^{2}\right)^{1 / 2}} \ln \frac{V+\left(1-p^{2} / k_{x}^{2}\right)^{1 / 2}}{V_{p} / k_{x}+\left(1-V^{2}\right)^{1 / 2}\left(1-p^{2} / k_{x}^{2}\right)^{1 / 2}}, \\
p<\left|k_{x}\right|, \\
1-\frac{V}{\left(p^{2} / k_{x}^{2}-1\right)^{1 / 2}}\left[\arcsin \frac{\left(p^{2} / k_{x}^{2}-1\right)^{1 / 2}}{\left(p^{2} / k_{x}^{2}-1+V^{2}\right)^{1 / 2}}\right. \\
\quad-\arcsin \frac{\left(1-V^{2}\right)^{1 / 2}\left(p^{2} / k_{x}-1\right)^{1 / 2}}{\left(p^{2} / k_{x}^{2}-1+V^{2}\right)^{1 / 2}}, \quad p>\left|k_{x}\right| .
\end{array}\right.
$$

At $p=+0$, the function (43) is

$$
\begin{aligned}
S(p, V, \eta)= & 1-\frac{V}{2} \int_{-1}^{1} d x \frac{\operatorname{sgn}(V-\eta x)}{\left((V-\eta x)^{2}-1+x^{2}\right)^{1 / 2}} \theta((V \\
& \left.-\eta x)^{2}-1+x^{2}\right),
\end{aligned}
$$

where $\eta=k_{y} / k_{x}$. Recall that at $p \rightarrow \infty S$ equals 1 , whereas at $p=0$ it is smaller than unity and becomes negative at large $V$.

Looking for the poles of an integrand of Eq. (44) with real axis,

$$
p^{2}=k_{x}^{2}+k_{y}^{2}+S(p, V, \eta),
$$

we note that when $S_{0}=S(p \rightarrow+0)$ is negative, there always will be two roots $p_{1}>0$ and $p_{2}>0$ of (47) in the certain domain of $\mathbf{k}$. This is seen from the graphical solution of Eq. (47), as shown in Fig. 4. Therefore, in this domain of 
wave vectors there will not exist any regular solution for the electric field, and therefore there is a crisis of the ThomasFermi screening. Let us specify the domain of the latter.

Evaluation of $S_{0}(V, \eta)$ gives

$S_{0}$

$$
=\left\{\begin{array}{l}
1-\frac{V}{2\left(1+\eta^{2}\right)^{1 / 2}} \ln \left|\frac{1-\alpha+\left((1-\alpha)^{2}-\beta^{2}\right)^{1 / 2}}{1+\alpha-\left((1+\alpha)^{2}-\beta^{2}\right)^{1 / 2}}\right|, \\
V>\eta \\
1-\frac{V}{2\left(1+\eta^{2}\right)^{1 / 2}} \ln \\
\times \frac{\beta^{2}}{\left[1+\alpha-\left((1+\alpha)^{2}-\beta^{2}\right)^{1 / 2}\right]\left[1-\alpha+\left((1-\alpha)^{2}-\beta^{2}\right)^{1 / 2}\right]}, \\
V<\eta,
\end{array}\right.
$$

where

$$
\alpha=V \frac{\eta}{1+\eta^{2}}, \quad \beta^{2}=\frac{1+\eta^{2}-V^{2}}{\left(1+\eta^{2}\right)^{2}}, \quad \eta=\frac{k_{y}}{k_{x}} .
$$

The function $S_{0}(V, \eta)$ for different $\eta$ is shown in Fig. 5. The smallest value of $V$ at which $S_{0}$ is negative is achieved at $\eta=0$, where

$$
S_{0}(V, 0)=1-\frac{V}{2} \ln \left|\frac{1+V}{1-V}\right| .
$$

This expression is negative at $V>V_{c}$ where $V_{c}$ $=0.8335$ is the solution of an equation

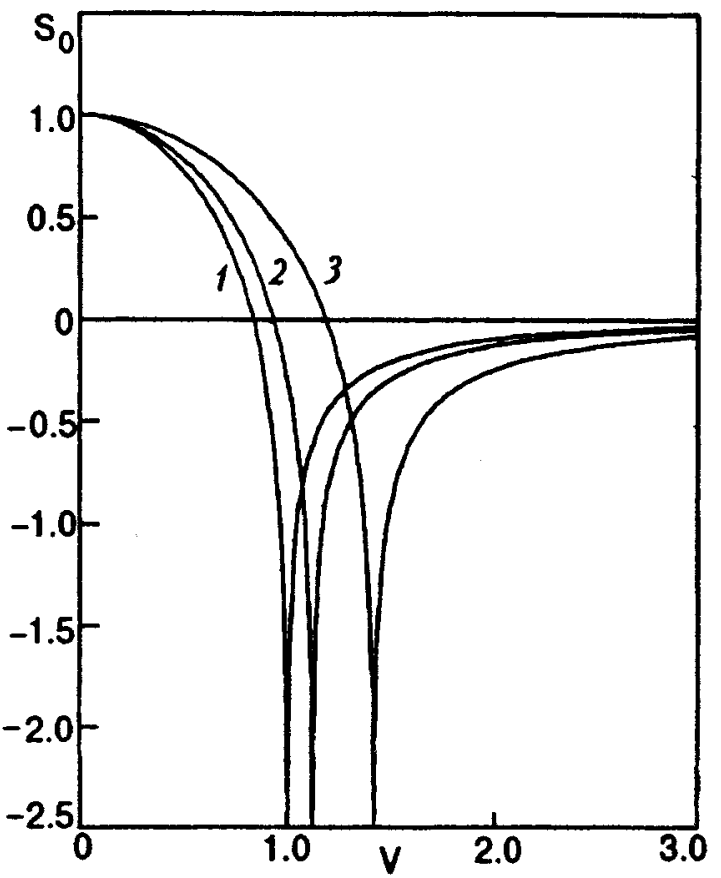

FIG. 5. Dependence of $S_{0}$ on $V$. Curves 1,2 , and 3 correspond to $\eta=0$, 0.5 , and 1.0 , respectively.

$$
V_{c}=\tanh \frac{1}{V_{c}} \text {. }
$$

Therefore, the instability of laminar flow occurs in a threedimensional metal at a velocity slightly smaller than the Fermi velocity. Near the critical value of $V$, the instability takes place at a small $k_{y}$-to- $k_{x}$ ratio. The smaller is $\left|k_{y}\right|$, the stronger is the distortion from the unperturbed $\phi(z)$ distribution. In effect, the large- $k_{y}$ Fourier components of the potential are virtually unaffected, whereas small the components are depressed. This implies a change of the potential and of the charge distribution inside a metal, which is shown schematically in Fig. 6. The shape of the image of (symmetrical) external charge in the surface sheet is compressed in the direction perpendicular to the direction of motion and is elongated in the opposite direction. At the same time, the penetration depth of electric field inside the metal increases. Near the critical velocity, the characteristic compression is

$$
\Delta z-\frac{\lambda_{T F}}{\left(V_{c}-V\right)^{1 / 2}}
$$

The effect of potential redistribution strongly manifests itself if the distance between the tip and the metal is of the order of a few unperturbed Thomas-Fermi screening lengths.

Let us analyze the analytical properties of $S$ in the complex plane $p . S(p)$ has a singularity along the imaginary axis $p=i q$, which is in effect a manifestation of the existence of the branching points of two-dimensional $S$ [Eq. (37)]. In a three-dimensional metal, maximal velocity of electron motion parallel to the metal surface $V_{\|}=\sin \theta$ may be smaller than 1 at $V<1$ in some range of $\theta$. The function $S(i q)$ attains different values when the imaginary axis is approached from the left and from the right, and remains analytical in the subspaces $\operatorname{Re} p<0$ and $\operatorname{Re} p>0$. The values of $S(p)$ to the left and to the right of the imaginary axis are

$$
\begin{aligned}
& S_{ \pm}(i q)=1+\frac{V}{2} \int_{0}^{\pi} d \theta R_{ \pm}\left(v, q / k_{x}\right), \\
& v=\frac{V-\eta \cos \theta}{\sin \theta},
\end{aligned}
$$

where $\eta=k_{y} / k_{x}$, and
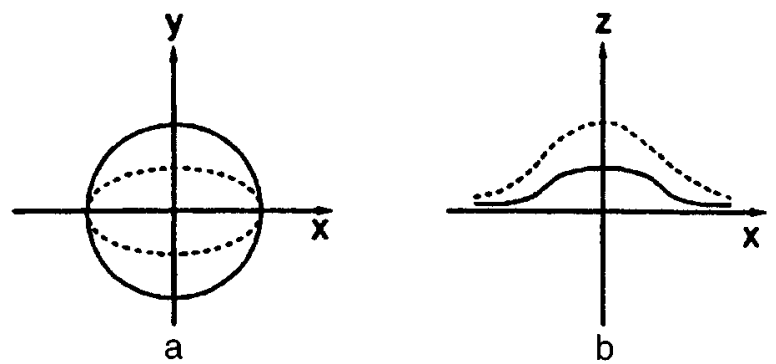

FIG. 6. Schematic diagram of the charge penetration inside a metal along the metal surface (a) and along the cross-sectional plane (b). Solid lines correspond to $V<V_{c}$, and dotted lines correspond to $V>V_{c}$. 


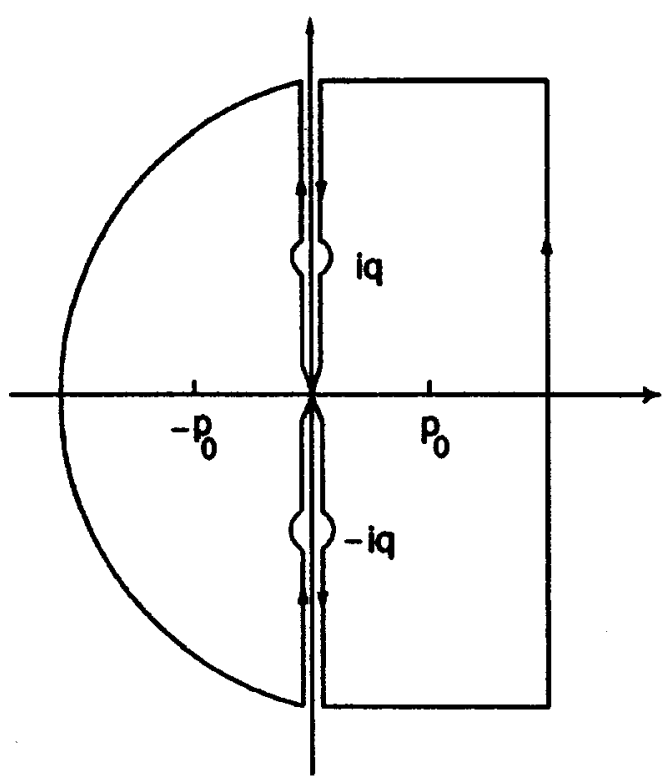

FIG. 7. Contour of integration for the calculation of the potential $\phi(z)$ [Eq. (24)] in a three-dimensional metal.

$$
R_{ \pm}(v)= \begin{cases}0, & |v|<1 \\ -\frac{\operatorname{sgn}(v)}{\left(v^{2}-1-x^{2}\right)^{1 / 2}}, & |v|>1,|x|<\sqrt{v^{2}-1} \\ \pm \frac{i \operatorname{sgn}(v x)}{\left(x^{2}-v^{2}+1\right)^{1 / 2}}, & |v|>1,|x|>\sqrt{v^{2}-1}\end{cases}
$$

where $x=q / k_{x}$.

We can now calculate from (24) the potential $\phi(z)$. Integrating along the path shown in Fig. 7, we obtain

$$
\begin{aligned}
\phi(z)= & \phi_{0} \exp \left(-p_{0} z\right)+\int \frac{d \Omega}{4 \pi} Z_{\varphi} \exp \left(-i \gamma_{\varphi} z\right) \\
& +\int_{-\infty}^{\infty} \frac{d q}{2 \pi} X_{q} \exp (i q z),
\end{aligned}
$$

where, as follows from the requirement that $\phi(z)$ vanish at $z \rightarrow \infty$, a relation between $\phi(0)$ and $\phi^{\prime}(0)$ is

$$
\phi^{\prime}(0)=-p_{0} \phi(0)-\int \frac{d \Omega}{4 \pi} \frac{A_{\varphi}}{p_{0}+i \gamma_{\varphi}} .
$$

The coefficients $\phi_{0}, Z_{\varphi}$, and $X_{q}$ in the expression (55) are

$$
\begin{aligned}
\phi_{0}= & \frac{2 p_{0}}{D^{\prime}\left(p_{0}\right)}\left[\phi(0)+\int \frac{d \Omega}{4 \pi} \frac{A_{\varphi}}{p_{0}^{2}+\gamma_{\varphi}^{2}}\right], \\
Z_{\varphi}= & \frac{1}{2}\left[\frac{1}{D_{+}\left(i \gamma_{\varphi}\right)}+\frac{1}{D_{-}\left(i \gamma_{\varphi}\right)}\right] A_{\varphi}, \\
X_{q}= & \left(\frac{1}{D_{+}\left(i \gamma_{\varphi}\right)}-\frac{1}{D_{-}\left(i \gamma_{\varphi}\right)}\right)\left[\left(i q-p_{0}\right) \phi(0)\right. \\
& \left.-\int \frac{d \Omega}{4 \pi} \frac{q+i p_{0}}{\left(p_{0}+i \gamma_{\varphi}\right)\left(q+\gamma_{\varphi}\right)}\right] A_{\varphi},
\end{aligned}
$$

where $D(p)=p^{2}-\mathbf{k}^{2}-S(p)$ is the denominator of an integrand of Eq. (24), which is appropriate for the $3 d$ case.
Proceeding further in the same manner as in Sec. 2, we calculate with the help of Eq. (55) the function $u_{\varphi}$

$$
\begin{aligned}
u_{\varphi}= & A_{\varphi} \exp \left(-i \gamma_{\varphi} z\right)+\frac{k_{x} V}{\sin \theta \sin \varphi}\left[\frac{\phi_{0}}{\gamma_{\varphi}+i p_{0}}\right. \\
& \times\left(\exp \left(-p_{0} z\right)-\exp \left(-i \gamma_{\varphi} z\right)\right) \\
& +\int \frac{d \Omega^{\prime}}{4 \pi} Z_{\varphi^{\prime}} \frac{\exp \left(-i \gamma_{\varphi^{\prime}} z\right)-\exp \left(-i \gamma_{\varphi^{\prime}} z\right)}{\gamma_{\varphi^{\prime}}-\gamma_{\varphi^{\prime}}} \\
& \left.+\int_{-\infty}^{\infty} \frac{d q}{2 \pi} X_{q} \frac{\exp (i q z)-\exp \left(-i \gamma_{\varphi} z\right)}{q+\gamma_{\varphi}}\right],
\end{aligned}
$$

where $A_{\varphi}$ is an arbitrary constant. Requiring that the terms proportional to $\exp \left(-i \gamma_{\varphi} z\right)$ cancel each other out at $\varphi>0$, and using, at $\varphi<0$, the boundary condition (42), we obtain

$$
\begin{aligned}
A_{\varphi}= & \frac{k_{x} V}{\sin \theta \sin \varphi}\left[\frac{\phi_{0}}{\gamma_{\varphi}+i p_{0}}+\int \frac{d \Omega^{\prime}}{4 \pi} \frac{Z_{\varphi^{\prime}}}{\gamma_{\varphi}-\gamma_{\varphi^{\prime}}}\right. \\
& \left.+\int_{-\infty}^{\infty} \frac{X_{q}}{q+\gamma_{\varphi}}\right]
\end{aligned}
$$

at $-\pi<\varphi<0$, and

$$
\begin{aligned}
A_{-\varphi} & =(1-q) A_{\varphi}+\frac{q}{\pi} \int_{0}^{\pi} \sin \theta d \theta \int_{0}^{\pi} d \varphi A_{\varphi} \sin \theta \sin \varphi \\
& =\hat{L} A_{\varphi}
\end{aligned}
$$

at $0<\varphi<\pi$, where $\hat{L}$ is a three-dimensional operator of a diffuse reflection, which can be written in the form

$$
\hat{L}=1-q+\hat{q},
$$

where $\hat{q}$ is an operator

$$
\hat{q} A_{\varphi}=\frac{q}{\pi} \int d \Omega_{+} A_{\varphi} \sin \theta \sin \varphi
$$

$\left(d \Omega_{+}\right.$means a solid-angle integration with a positive $\left.\varphi\right)$. It follows also that the inverse operator is

$$
\hat{L}^{-1}=\frac{1-\hat{q}}{1-q} .
$$

Combining Eqs. (57) and (60), we obtain an integral equation for $A_{\varphi}$ in the domain $0<\varphi<\pi$

$$
\begin{aligned}
\hat{L} A_{\varphi} & -\frac{k_{x} V}{\sin \theta \sin \varphi}\left\{\int \frac{d \Omega_{+}^{\prime}}{8 \pi}\left(\frac{A_{\varphi^{\prime}}}{\gamma_{\varphi}+\gamma_{\varphi^{\prime}}}+\frac{\hat{L} A_{\varphi^{\prime}}}{\gamma_{\varphi}-\gamma_{\varphi^{\prime}}}\right)\right. \\
& \times\left(\frac{1}{D_{+}\left(i \gamma_{\varphi}\right)}+\frac{1}{D_{-}\left(i \gamma_{\varphi}\right)}\right) \\
& -\int_{-\infty}^{\infty} \frac{d q}{2 \pi} \frac{q+i p_{0}}{q-\gamma_{\varphi}} \int \frac{d \Omega_{+}^{\prime}}{4 \pi}\left[\frac{A_{\varphi^{\prime}}}{\left(p_{0}+i \gamma_{\varphi^{\prime}}\right)\left(q+\gamma_{\varphi^{\prime}}\right)}\right. \\
& \left.\left.\left.+\hat{L} \frac{A_{\varphi^{\prime}}}{\left(p_{0}-i \gamma_{\varphi^{\prime}}\right)\left(q-\gamma_{\varphi^{\prime}}\right)}\right]\right\}=\frac{k_{x} V_{0}}{\sin \theta \sin \varphi}\left[\frac{\phi_{\varphi_{\varphi}}-i p_{0}}{\gamma_{0}}\right)\right], \\
& \left.+\phi(0) \int_{-\infty}^{\infty} \frac{d q}{2 \pi} \frac{i q-p_{0}}{\gamma_{\varphi}-i q}\left(\frac{1}{D_{+}(i q)}-\frac{1}{D_{-}(i q)}\right)\right]
\end{aligned}
$$


where $D_{ \pm}(i q)$ is a value of $D(p)$ to the left/right of an imaginary axis $p=i q \pm 0$.

Equation (66) is valid at $V<V_{c}$ when the linear regime of the surface sheet motion is realized. In this case the solution for $A_{\varphi}$, together with Eq. (56), permits determination of the effective boundary condition, i.e., the value of the ratio $\phi^{\prime} / \phi$ at $z=0$.

\section{ENERGY DISSIPATION IN A MOVING SURFACE SHEET}

In this section we will consider the energy losses in a surface sheet as a result of its interaction with the external charge that pulls the sheet. The force acting on the sheet is

$$
\mathbf{F}=\mathbf{E} \rho,
$$

where the surface charge density $\rho$ is determined as $(1 / 4 \pi)$ $\times(\partial \phi / \partial z)_{z=0}$, and $E_{x}=-(\partial \phi / \partial z)_{z=0}$. The product $F_{x} V=W$ gives the power dissipated in a metal. Integrating with respect to space coordinates $x, y$ and performing the Fourier transformation, we obtain

$$
W=\frac{V}{4 \pi} \int \frac{d^{2} k}{(2 \pi)^{2}} k_{x} p_{0}(\mathbf{k})\left|\phi_{\mathbf{k}}(0)\right|^{2} \operatorname{Im} \zeta(\mathbf{k}),
$$

where $\mathbf{k}=\left(k_{x}, k_{y}\right)$. The quantity $\zeta(\mathbf{k})$ is the coefficient in the boundary condition at the metal surface

$$
\phi^{\prime}(0)=-p_{0}(1+\zeta(\mathbf{k})) \phi(0)
$$

[we dropped the index $\mathbf{k}$ in $\phi_{\mathbf{k}}(0)$ and $\phi_{\mathbf{k}}^{\prime}(0)$ ]. Using Eq. (56), we obtain

$$
\zeta(\mathbf{k})=\frac{1}{p_{0}} \int \frac{d \Omega_{+}}{4 \pi}\left(\frac{A_{\varphi}}{p_{0}+i \gamma_{\varphi}}+\frac{\hat{L} A_{\varphi}}{p_{0}-i \gamma_{\varphi}}\right) / \phi(0),
$$

where $A_{\varphi}$ is found from the integral equation (66).

In the case of absence of a $y$-dependence of the potential (for example, for an infinite rod moving parallel to the surface), an expression for the rate of the energy dissipation per unit length is

$$
W=\frac{V}{4 \pi} \int \frac{d k_{x}}{2 \pi} k_{x} p_{0}\left(k_{x}\right)\left|\phi_{k_{x}}(0)\right|^{2} \operatorname{Im} \zeta\left(k_{x}\right),
$$

where $\zeta\left(k_{x}\right)$ is found by setting $k_{y}=0$ in (70). In the case of small $|\mathbf{k}|$, Eq. (66) can be solved iteratively in $k_{x}$ :

$$
A_{\varphi}=A_{\varphi}^{0}+k_{x} A_{\varphi}^{1}+\ldots .
$$

In the lowest approximation we obtain

$$
\begin{aligned}
\hat{L} A_{\varphi}= & \frac{k_{x} V}{\sin \theta \sin \varphi}\left[\frac{1}{\gamma_{\varphi}-i p_{0}}\right. \\
& \left.+\int_{-\infty}^{\infty} \frac{d q}{2 \pi} \frac{p_{0}-i q}{q-\gamma_{\varphi}}\left(\frac{1}{D_{+}(i q)}-\frac{1}{D_{-}(i q)}\right)\right] \phi(0),
\end{aligned}
$$

where $\gamma_{\varphi}$ is determined in (41) with $\mathrm{v}=+0$. Typical values of $q$ are on the order of $k_{x}$, i.e., much smaller than the inverse Thomas-Fermi screening length $\kappa_{T F}$ (in the dimensionless units we have $\left.\left|k_{x}\right| \ll 1\right)$. We introduce the function

$$
R(q)=\frac{1}{2 i}\left(\frac{1}{D_{+}(i q)}-\frac{1}{D_{-}(i q)}\right),
$$

where

$$
D_{ \pm}(i q)=-\mathbf{k}^{2}-q^{2}-S_{ \pm}(i q) ; \quad S_{ \pm}(i q)=S(i q \pm 0) .
$$

Setting $S_{ \pm}(i q)=S_{1}(q) \pm i S_{2}(q)$, we obtain from (53)

$$
\begin{aligned}
S_{1}(q)= & 1-\frac{V}{2} \int_{-1}^{1} d x \frac{\operatorname{sgn}(V-\eta x)}{\left(\Lambda(x)-q^{2} / k_{x}^{2}\right)^{1 / 2}} \theta(\Lambda(x) \\
& \left.-q^{2} / k_{x}^{2}\right), \\
S_{2}(q)= & \frac{V}{2} \int_{-1}^{1} d x \frac{\theta(\Lambda(x)) \operatorname{sgn}(V-\eta x)}{\left(q^{2} / k_{x}^{2}-\Lambda(x)\right)^{1 / 2}} \theta\left(\frac{q^{2}}{k_{x}^{2}}\right. \\
& -\Lambda(x)) \operatorname{sgn}\left(\frac{q}{k_{x}}\right),
\end{aligned}
$$

where $x=\cos \theta$, and $\Lambda(x)=(V-\eta x)^{2}+x^{2}-1$. At $\eta=0$, a direct integration gives the following expression for the positive values of $q$ and $k_{x}$ :

$$
S_{1}(q)= \begin{cases}1-V \ln \frac{1+\left(V^{2}-q^{2} / k_{x}^{2}\right)^{1 / 2}}{\left(1-V^{2}+q^{2} / k_{x}^{2}\right)^{1 / 2}}, & q / k_{x}<V, \\ 1, & q / k_{x}>V\end{cases}
$$

and

$$
S_{2}(q)=\left\{\begin{array}{cc}
V\left(\frac{\pi}{2}-\arcsin \frac{\left(1-V^{2}\right)^{1 / 2}}{\left(1-V^{2}+q^{2} / k_{x}^{2}\right)^{1 / 2}}\right), & q / k_{x}<V \\
V\left(\arcsin \frac{1}{\left(1-V^{2}+q^{2} / k_{x}^{2}\right)^{1 / 2}}\right. & \\
-\arcsin \frac{\left(1-V^{2}\right)^{1 / 2}}{\left(1-V^{2}+q^{2} / k_{x}^{2}\right)^{1 / 2}}, & q / k_{x}>V .
\end{array}\right.
$$

The dependences $S_{1,2}(q)$ at various $V$ and $\eta$ are shown in Fig. 8. An approximate value of $R(q)$ at $\left|k_{x, y}\right| \ll 1$ is

$$
R(q) \approx \frac{S_{2}(q)}{S_{1}^{2}(q)+S_{2}^{2}(q)} .
$$

$R(q)$ is an odd function of $q$, which vanishes linearly at small $\left|q / k_{x}\right|$ and which behaves at $1 / q$ at $|q| \gg\left|k_{x}\right|$.

The two terms on the right side of Eq. (73) represent the contributions to the dissipation emerging from the main pole $p=p_{0}$ in the complex plane $p$, and from the branching point along the imaginary axis. The contributions to $\zeta(\mathbf{k}), \zeta_{1}(\mathbf{k})$, and $\zeta_{2}(\mathbf{k})$ prove to be of the same order of magnitude. Substitution of Eq. (73) into Eq. (70) at $p_{0} \approx 1$ and small $k_{x}$ [see Eq. (36)] gives

$$
\begin{aligned}
\operatorname{Im} \zeta_{1}(\mathbf{k})= & \frac{k_{x} V}{1-q} \int \frac{d \Omega_{+}}{4 \pi} \frac{2-q-q \gamma_{\varphi}^{2}}{\left(1+\gamma_{\varphi}^{2}\right)^{2} \sin \theta \sin \varphi} \\
& -\frac{k_{x} V}{1-q} \int \frac{d \Omega_{+}}{4 \pi} \frac{1}{1+\gamma_{\varphi}^{2}} \int \frac{d \Omega_{+}}{\pi} \frac{q}{1+\gamma_{\varphi}^{2}} \\
& +\frac{k_{x} V}{1-q} \int \frac{d \Omega_{+}}{4 \pi} \frac{\gamma_{\varphi}}{1+\gamma_{\varphi}^{2}} \int \frac{d \Omega_{+}}{\pi} \frac{q \gamma_{\varphi}}{1+\gamma_{\varphi}^{2}},
\end{aligned}
$$



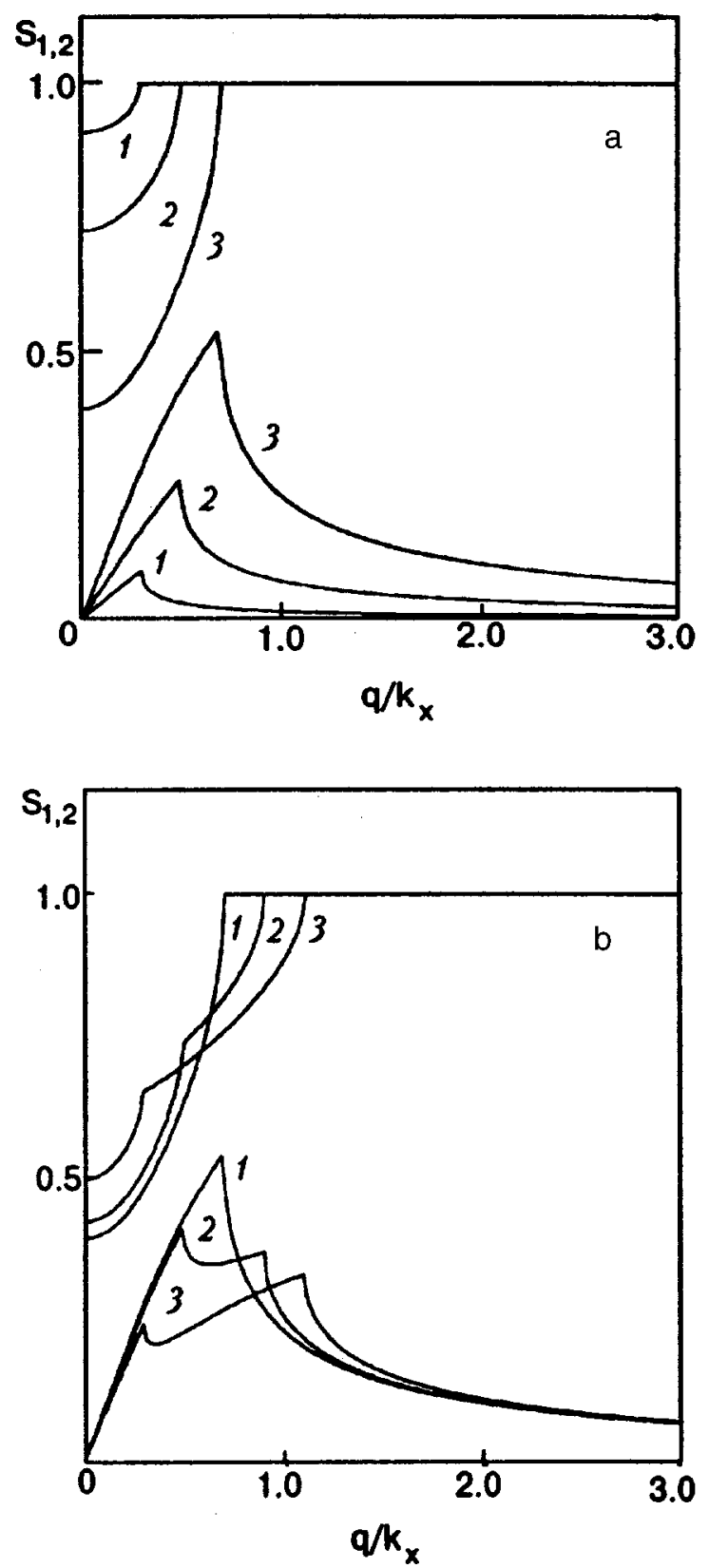

FIG. 8. Dependences of $S_{1}$ (upper curves) and $S_{2}$ (lower curves) on $q$. (a) $\eta=0$. Curves 1,2 , and 3 correspond to $V=0.3,0.5$, and 0.7 ; (b) $V$ $=0.7$. Curves 1,2 , and 3 correspond to $\eta=0,0.2$, and 0.4 .

$$
\begin{aligned}
\operatorname{Im} \zeta_{2}(\mathbf{k})= & \frac{k_{x} V}{1-q} \int \frac{d \Omega_{+}}{4 \pi} \\
& \times \frac{(2-q) R_{0}(q)-q \gamma_{\varphi} R_{1}\left(\gamma_{\varphi}\right)-\gamma_{\varphi} R\left(\gamma_{\varphi}\right)}{\left(1+\gamma_{\varphi}^{2}\right) \sin \theta \sin \varphi} \\
& +\frac{k_{x} V}{1-q} \int \frac{d \Omega_{+}}{4 \pi} \frac{1}{1+\gamma_{\varphi}^{2}} \int \frac{d \Omega_{+}}{\pi} \\
& \times\left[-q R_{0}\left(\gamma_{\varphi}\right)+\frac{q}{2} \gamma_{\varphi} R\left(\gamma_{\varphi}\right)\right] \\
& +\frac{k_{x} V}{1-q} \int \frac{d \Omega_{+}}{4 \pi} \frac{\gamma_{\varphi}}{1+\gamma_{\varphi}^{2}} \int \frac{d \Omega_{+}}{\pi}
\end{aligned}
$$

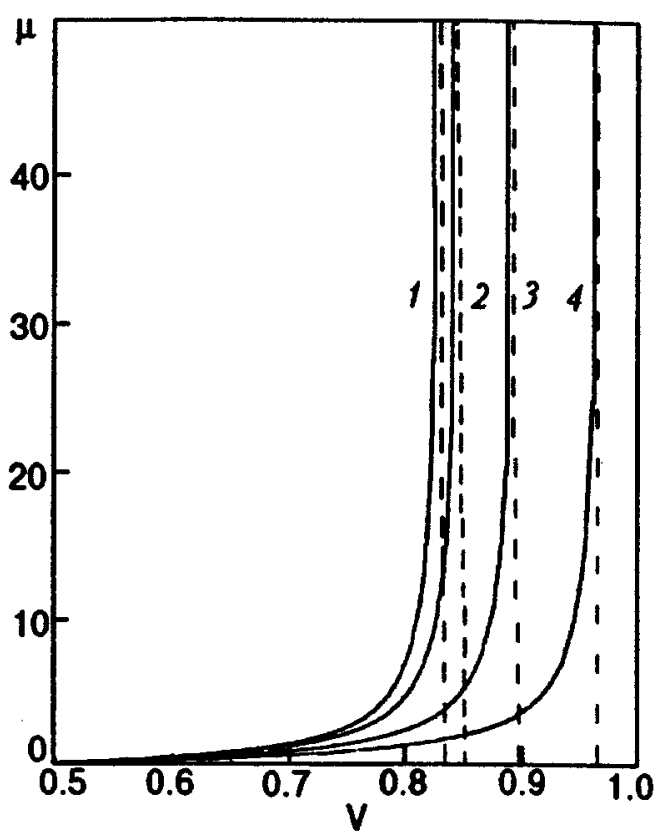

FIG. 9. Dependence of $\mu$ on $V$. Curves $1,2,3$, and 4 correspond to $\eta$ $=0,0.2,0.4$ and 0.6 .

$$
\times\left[q R_{1}\left(\gamma_{\varphi}\right)+\frac{q}{2} R\left(\gamma_{\varphi}\right)\right]
$$

where

$$
R_{n}(x)=\frac{1}{\pi} \mathrm{V} \cdot \mathrm{p} \cdot \int_{-\infty}^{\infty} \frac{R(q) q^{n}}{q-x} d q .
$$

Inspection of integrals in Eqs. (81) and (82) shows that at $k_{x} \rightarrow 0 \int d \Omega_{+} /\left(1+\gamma_{\varphi}^{2}\right)$ takes a constant value, whereas $\int d \Omega_{+} \gamma_{\varphi} /\left(1+\gamma_{\varphi}^{2}\right)$ behaves as $k_{x} \ln \left(1 / k_{x}\right)$. This means that the last term in Eq. (82) can be ignored at small value of $\mathbf{k}$. The second term is of the order of $k_{x}$, whereas the first term behaves as $k_{x} \ln \left(1 / k_{x}\right)$.

For orientation, we assume that $R(q)$ is $C q /\left(q^{2}\right.$ $\left.+a^{2}\right)$, which gives from Eq. (81) $R_{0}(q)=C a /\left(q^{2}\right.$ $\left.+a^{2}\right)$ and $R_{1}(q)=-C a q /\left(q^{2}+a^{2}\right)$. One can then evaluate integrals in (82). It appears that the last term in this expression is of the same order of magnitude as the corresponding term in Eq. (81); therefore, it can be ignored. The second term in Eq. (82) is proportional to $k_{x} \ln \left(1 / k_{x}\right)$. Evaluation of the leading (logarithmic) term in $\zeta_{2}$ requires the knowledge of the functions $R_{0,1}$ at $q=0$. After some algebra, we obtain

$$
\operatorname{Im} \zeta(\mathbf{k})=k_{x} V \frac{1-q / 2}{1-q}\left[\ln \frac{C_{1}}{k_{x}}+\mu \ln \frac{C_{2}}{k_{x}}\right],
$$

where $C_{1,2} \sim 1$ are complex functions of $V, \eta$, and $q$ and $\mu$ is a quantity

$$
\mu=\frac{2}{\pi} \int_{0}^{\infty} \frac{R(x)}{x} d x
$$

which is shown for different values $V$ and $\eta$ in Fig. 9 .

Since $\zeta$ is a small quantity $(|\zeta| \ll 1)$, the field outside the metal is almost equal to its value calculated for an ideally 


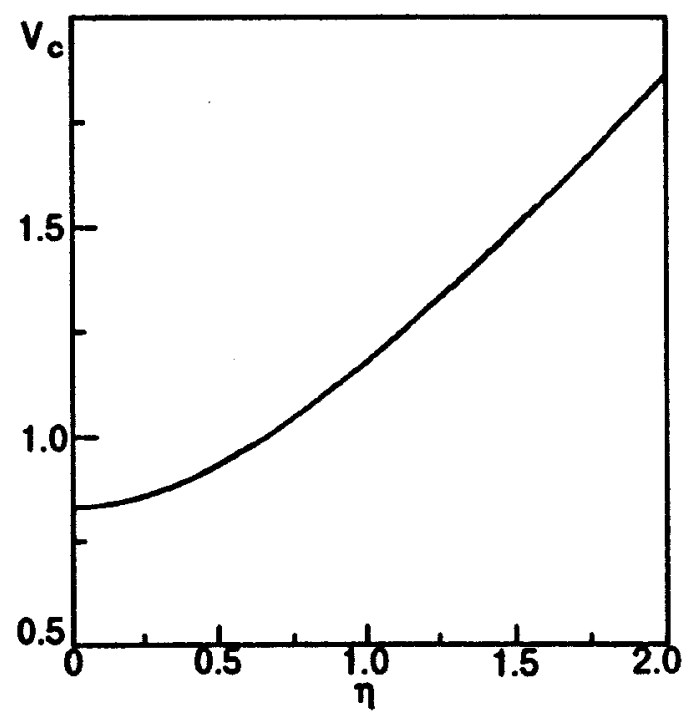

FIG. 10. Dependence of $V_{c}$ on $\eta$.

reflecting metallic surface $\left(\lambda_{T F}=0\right)$. The power dissipated due to the tip motion becomes (in the dimensionless units)

$$
W \simeq \frac{V}{4 \pi} \int \frac{d^{2} k}{(2 \pi)^{2}} k_{x} \frac{\left|\phi_{\mathbf{k}}^{\prime}(0)\right|^{2}}{p_{0}(\mathbf{k})} \operatorname{Im} \zeta(\mathbf{k}) .
$$

In the dimensional units, the dissipated power is

$$
\begin{aligned}
W= & \frac{V^{2}}{4 \pi e^{2} N\left(\varepsilon_{F}\right) V_{F}} \frac{1-q / 2}{1-q} \\
& \times \int \frac{d^{2} k}{(2 \pi)^{2}} k_{x}^{2}\left|\phi_{\mathbf{k}}^{\prime}(0)\right|^{2} \\
& \times\left[\ln \frac{\widetilde{C}_{1}}{\left|k_{x}\right| \lambda_{T F}}+\mu \ln \frac{\widetilde{C}_{2}}{\left|k_{x}\right| \lambda_{T F}}\right] .
\end{aligned}
$$

Assuming that the tip is a point charge $Q$, we obtain an estimate of $W$ valid at $V \ll V_{c}$

$$
W_{1} \simeq \frac{V^{2} Q^{2} \lambda_{T F}^{2}}{V_{F} d^{4}} \frac{1-q / 2}{1-q},
$$

where $d$ is a distance between the tip and the metal surface. At small $d \simeq \lambda_{T F}$, this expression matches in order of magnitude the loss of a charged particle that moves inside a metal.

For a charged rod with a charge $Q$ per unit length, an estimate of the loss per unit length is

$$
W_{2} \sim \frac{V^{2} Q^{2} \lambda_{T F}^{2}}{V_{F} d^{3}} \frac{1-q / 2}{1-q} .
$$

The quantity $\mu$ in (84) increases dramatically at $V$ near the critical velocity $V_{c}$. At a value of $V$ larger than $V_{c}$, the linear regime of the surface screening breaks down. An asymptotic behavior of $\mu$ near $V_{c}$

$$
\mu \simeq \frac{1}{|\mathbf{k}|^{2}+S_{0}(V, \eta)}, \quad|\mathbf{k}| \ll 1,
$$

where $S_{0} \rightarrow 0$ in the limit $V \rightarrow V_{c}(\eta)$. The function $V_{c}(\eta)$ is shown in Fig. 10.
Sharp resonances of $\mu$ versus $V$ occur at a fixed values of the momenta $k_{x}$ and $k_{y}$. Dissipated power $W$ can be determined by integration of $\mu$ in Eq. (85) with respect to $\mathbf{k}$. Whether the dissipated power $W$ vs $V$ will have similar sharp resonances depends on the actual potential distribution at the metal surface.

Let us consider as an example a point charge $Q$ at a height $h$ above the metal surface giving at $\lambda \rightarrow 0$

$$
\begin{aligned}
\phi(\mathbf{r})= & Q\left(\frac{1}{\left(x^{2}+y^{2}+(z-h)^{2}\right)^{1 / 2}}\right. \\
& \left.-\frac{1}{\left(x^{2}+y^{2}+(z+h)^{2}\right)^{1 / 2}}\right),
\end{aligned}
$$

from which we have

$$
\phi_{\mathbf{k}}^{\prime}(0)=4 \pi Q \exp (-2|\mathbf{k}| h)
$$

and an infinite thin rod with the linear charge density $Q$, for which

$$
\phi(\mathbf{r})=\frac{Q}{2} \ln \frac{x^{2}+(z-h)^{2}}{x^{2}+(z+h)^{2}}
$$

and, correspondingly

$$
\phi_{\mathbf{k}_{x}}^{\prime}(0)=2 \pi Q \exp \left(-2\left|k_{x}\right| h\right) .
$$

In the second case we then obtain

$$
W \sim \frac{W_{2}(V)}{S_{0}(V, 0)+1 / 4 h^{2}}, \quad h \gg 1,
$$

and in the first case

$$
W \sim W_{1}(V) \int_{0}^{\infty} \frac{d \eta}{\left(1+\eta^{2}\right)^{2}} \frac{1}{S_{0}(V, \eta)+\left(1+\eta^{2}\right) / 4 h^{2}} .
$$

The dependences (95) and (96) are shown in Fig. 11.

\section{DISCUSSION}

Dynamical interaction of a moving charge with a metal surface reveals singularities in the dissipated power as a function of the velocity of motion $V$. Depending on the topology of the Fermi surface, the maximum of power dissipation in the surface sheet occurs either at the Fermi velocity or slightly below it. At the same value of $V$, the electric field begins penetrating the metal to a depth much greater than the Thomas-Fermi length, thus breaking the Thomas-Fermi screening barrier.

Crucial for the observation of such effects is the possibility of realization of fast motion of a surface charge. This can be achieved by propagating charged particles or small charged bodies above and near the metal surface. The other possibility may be in creating an electronically driven motion of a surface charge parallel to the metal surface. Concerning the latter, we envisage a setup with an array of equally spaced metallic electrodes near the bulk metal (Fig. 12a) biased periodically in time with the short electric pulses of fixed polarity. This will create maxima in the surface charge distribution in a metal moving between subsequent locations in the metal surface with an average velocity $\bar{V}$ $=\Delta x / \Delta t(\Delta x$ is the distance between electrodes, and $\Delta t$ is 


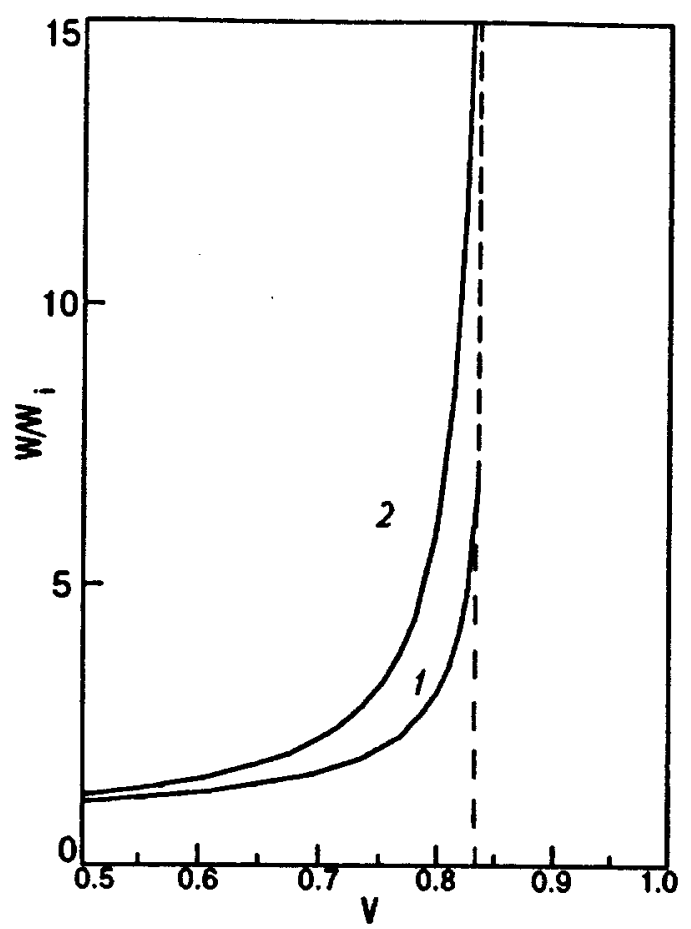

FIG. 11. Normalized dissipation $W / W_{1}$ (curve 1 ) and $W / W_{2}$ (curve 2) as a function of the velocity $V$ at $h=2.5$.

the interval between pulses). The velocity of the order of the Fermi velocity $V_{F} \sim 10^{8}-10^{6} \mathrm{~cm} / \mathrm{s}$ can be easily obtained with the corresponding choice of $\Delta x$ and $\Delta t$.

The other possibility is a motion of a charged soliton of some kind in a semiconducting or a superconducting film overlaying the metal (Fig. 12b). For instance, in the case of the Gunn effect in semiconductors, a moving charged soliton is formed due to an $\mathrm{N}$-shaped current-voltage characteristic of the semiconductor. ${ }^{14}$ The size of the soliton in GaAs is of
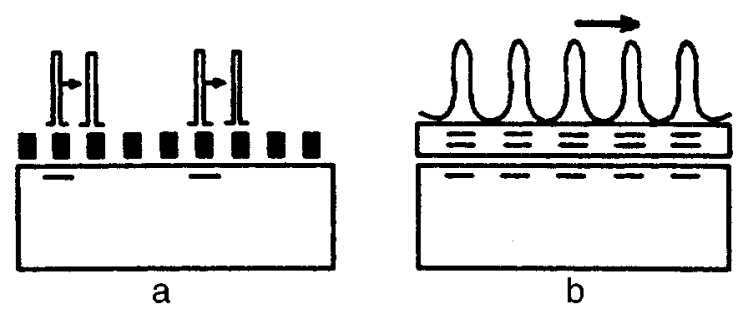

FIG. 12. Schematic diagram of the electronically driven motion of a surface charge. (a) Electric pulses switched periodically between metallic electrodes near the metal surface; (b) Propagating solitons in the semiconductor layer overlaying the bulk metal. the order of $10 \mu \mathrm{m}$. The velocity of soliton motion can be made quite large, $V \sim 10^{7} \mathrm{~cm} / \mathrm{s}$. As a result of the interaction of solitons with the induced surface charges in a metal, the current-voltage characteristic of a semiconductor film overlaying the metal attains a singularity at $V$ near the Fermi velocity of the metal.

Another possibility is propagating low-frequency charged plasmons ${ }^{15-17}$ in a thin superconducting film in the vicinity of a bulk metallic electrode.

It should be noted that the effect considered in this paper, an additional dissipation related to the surface charge, may have relevance to an evaluation of the quality factor $Q_{f}$ of an rf cavity, in particular, a superconducting cavity. At the lowest temperature at which the power absorption due to the electronic excitations in a superconductor is quite small (and, therefore, $Q_{f}$ large), a dissipation related to the surface charge may contribute to the residual value of $Q_{f}$ attained at the lowest temperature in a very high-quality cavities $\left(Q_{f}\right.$ $\left.\sim 10^{10}\right){ }^{18}$

*E-mail:kulik@fen.bilkent.edu.tr

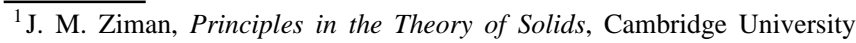
Press, Cambridge (1972).

${ }^{2}$ D. Pines, Elementary Excitations in Solids, Benjamin, New York (1964).

${ }^{3}$ M. H. Day, Phys. Rev. B12, 514 (1975).

${ }^{4}$ M. Day and M. Ebel, Phys. Rev. B19, 3434 (1979).

${ }^{5}$ Scanning Tunneling Microscopy and Related Methods, R. J. Behm, N. Garcia, and H. Rohrer (Eds.), Kluwer, Deventer (1990).

${ }^{6}$ D. V. Averin and K. K. Likharev, in Mesoscopic Phenomena in Solids, p. 173, B. L. Altshuler, P. A. Lee, and R. A. Webb (Eds.), North-Holland, Amsterdam (1991).

${ }^{7}$ I. K. Yanson, Fiz. Nizk. Temp. 9, 676 (1983) [Sov. J. Low Temp. Phys. 9, 343 (1983)].

${ }^{8}$ I. O. Kulik, Fiz. Nizk. Temp. 18, 450 (1992) [Sov. J. Low Temp. Phys. 18, $302(1992)]$

${ }^{9}$ I. O. Kulik, Physica B, Cond. Matter (Amsterdam) 218, 252 (1996).

${ }^{10}$ E. M. Lifshitz and L. P. Pitaevskil, Theoretical Physics, Vol. X. [Fizicheskaya Kinetika (Physical Kinetics), Nauka Publ., Moscow (1979)].

${ }^{11}$ P. M. Echenique, F. Flores, and R. H. Ritchie, in Solid State Physics: Advances in Research and Applications 43, p. 229, H. Ehrenreich and D. Turnbull (Eds.), Academic Press, New York (1990).

${ }^{12}$ A. A. Abrikosov, Fundamentals of the Theory of Metals, North-Holland, Amsterdam (1988).

${ }^{13}$ L. D. Landau and E. M. Lifshitz, Electrodynamics of Continuous Media, Pergamon, Press, Oxford (1984).

${ }^{14}$ B. K. Ridley, in Negative Differential Resistance and Instabilities in $2 D$ Semiconductors, N. Balkan, B. K. Ridley, and A. J. Vickers (Eds.), Plenum Press, New York (1993).

${ }^{15}$ I. O. Kulik, Zh. Éksp. Teor. Fiz. 65, 2016 (1073) [Sov. Phys. JETP 38, 1008 (1974)].

${ }^{16}$ J. E. Mooij and G. Schon, Phys. Rev. Lett. 55, 114 (1985).

${ }^{17}$ O. Bulsson, P. Xavier, and J. Richard, Phys. Rev. Lett. 73, 3153 (1994).

${ }^{18}$ D. M. Ginsberg and L. C. Hebel, In Superconductivity, Vol. 1, p. 193, R. D. Parks (Ed.), Marcel Dekker, New York (1969).

This article was published in English in the original Russian journal. It was edited by S. J. Amoretty. 